\title{
Diagnostic cross-sectional imaging of arterial diseases: really noninvasive?
}

\author{
Jean-Pierre Laissy $•$ Nicoletta Pasi $\cdot$ Jean-Michel Serfaty
}

Received: 6 August 2010 /Revised: 21 September 2010 / Accepted: 25 September 2010 /Published online: 15 November 2010

(C) European Society of Radiology 2010

Over the last 20 years, diagnostic cross-sectional imaging of arterial diseases has become less and less invasive, with the advent of MR angiography (MRA) and CT angiography (CTA). However, with the increasing proportion of elderly patients, some precautions are needed to avoid the two main complications of these techniques: development of nephrogenic systemic fibrosis with the former, and aggravation of a preexisting renal insufficiency with the latter. Moreover, CT angiography is inevitably associated with a high radiation dose that is problematic when younger patients need to be examined and monitored for various artery diseases (for example, patients with Marfan disease, Takayasu arteritis...). All the manufacturers have attempted during the 3 last years to find solutions in order to decrease potential hazards due to contrast medium administration with either CT or MR, and to reduce radiation dose with CT. Several scientific studies ensued or are under consideration for clinical validation.

For example, three recent major articles published during 2009-2010 show that it is possible to greatly reduce the risks of these techniques, by using unenhanced MRA on one hand $[1,2]$, and by reducing both the amount of contrast medium and radiation dose required for CTA on the other [3].

\section{Unenhanced MRA}

The first techniques to be employed were time-of-flight (TOF) and phase-contrast (PC) techniques, which allowed

J.-P. Laissy $(\bowtie) \cdot$ N. Pasi $\cdot$ J.-M. Serfaty

Department of Radiology, Hôpital Bichat APHP,

46 rue Henri Huchard,

75877, Paris Cedex 18, France

e-mail: jean-pierre.laissy@bch.aphp.fr images to be obtained selectively from either arteries or veins, by means of presaturation bands with the former, and various flow encoding directions with the latter. Nevertheless, these techniques had several limitations, the most important of them being long data acquisition times which often led to motion artefact. For example, these unenhanced MRA techniques such as twodimensional (2D) and three-dimensional (3D) time-offlight sequences were well suited to intracranial circulation, but very sensitive to flow-related artifacts and much too time consuming to image the entire thoracic or abdominal aorta [4]. Angiograms exhibiting high contrast between the vessels and surrounding tissues could not be consistently obtained due to flow and saturation effects within abdominal vessels (that course in different directions), and because of bowel peristalsis, respiratory and cardiac motion.

In a recent study by Krishnam et al. [1], focusing on the thoracic aorta, a steady state free precession (SSFP) T2 prep MR imaging sequence was used to achieve intense intravascular signal-to-noise ratio (SNR) for improved visualization of vascular structures [5-8].

The use of a free-breathing cardiac and respiratory gated 3D SSFP MRA sequence with non-selective radiofrequency (RF) excitation was expected to overcome documented pitfalls of 2D SSFP, such as artifacts due to sensitivity to magnetic B0 field heterogeneity, as thin-slice 2D SSFP requires longer TR/TE than possible with equivalent voxel resolution as with 3D SSFP [5-7]. This sequence was performed with a large field of view in the coronal plane in order to encompass the whole aorta in 50 consecutive patients with known or suspected thoracic aortic disease. To validate this new acquisition model, this unenhanced 3D SSFP angiographic sequence was compared with conventional contrast-enhanced (CE) MRA of thoracic aorta at $1.5 \mathrm{~T}$. 
All findings on SSFP MRA were confirmed on CEMRA in all patients. There were no false positive or negative SSFP MRA studies. Thus this new technique yielded $100 \%$ sensitivity, specificity, positive and negative predictive values, and diagnostic accuracy for the detection of aortic disease (admittedly using CE-MRA as the gold standard). Thus this technique shows great promise and may be an alternative non-invasive approach to assess or screen for thoracic aortic disease (Fig. 1) in selected patients at high risk of developing gadolinium-based contrast materialrelated complications, difficulty in breath-holding, difficult venous access, and in pregnant women without ionizing radiation exposure nor intravenous contrast material.

Because this new technique offers so much promise, all the major companies have endeavoured to find the best solution to obtain accurate MR angiograms without use of contrast medium for all regions of the body. For the diagnosis of renal artery stenosis, Toshiba was the first to implement the FBI (fresh blood imaging) acquisition, followed by the other constructors who developed other types of sequences with various acronyms.

MR angiography of the lower limbs is more challenging, since the coverage volume is important and the calibre of the arteries studied varies from $8 \mathrm{~mm}$ on average at the level of iliac arteries to $1 \mathrm{~mm}$ at the level of plantar arch.

Gjesdal et al. [2] also used a balanced non-SSFP sequence which seems to be very promising. This sequence provides high blood-to-muscle contrast which, in combination with fat suppression, makes peripheral unenhanced MR angiography feasible (eg of the lower limbs). The optimized MR technique is a 3D multi-shot balanced transient field echo sequence with fat suppression, which allows to selectively enhance arteries and veins. High signal from free fluid due to the T2 effects of this sequence is removed by applying an inversion prepulse with the appropriate inversion time or with the use of postprocessing tools, at the expense of lower image quality. The optimization of this sequence was performed in three healthy volunteers. Six different stations in the axial plane were obtained from the pelvic floor to the feet, resulting in a total examination time of about $15 \mathrm{~min}$. The major limitation of this sequence in its present form is that it cannot discriminate between arteries and veins. Ongoing improvements remain necessary.

\section{Low-radiation CTA with reduced dose of contrast agent}

With the advent of 64 and more detector systems, the amount of iodinated contrast medium has dramatically been reduced. Simultaneously, many technological solutions have been proposed to reduce radiation dose for $\mathrm{CT}$ angiography.

The study of Utsunomiya et al. [3] highlights the use of CTA protocols using $80 \mathrm{kVp}$ and $1.2 \mathrm{ml} / \mathrm{kg}$ iodine in
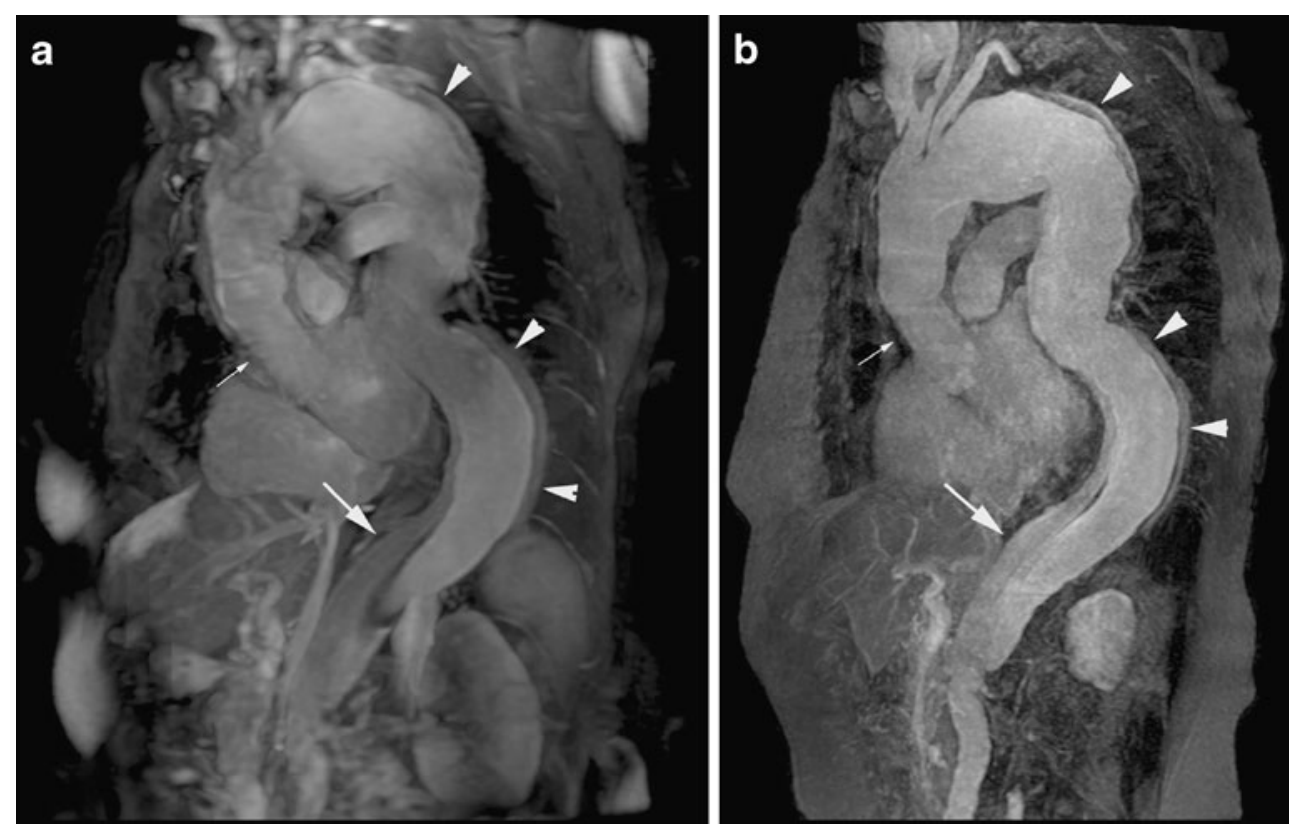

Fig. 1 Post-surgical MR imaging of type A dissection with aneurysmal dilation and residual intimal flap in the arch and descending aorta. Sagittal oblique unenhanced 3D SSFP MRA image (a) and corresponding sagittal oblique CE-MRA image (b) both demonstrate the ascending aortic surgical graft (small arrow), mural thrombus in the posterior false lumen of the aortic arch and descending aorta (arrowheads), aneurysmal dilatation of the aortic arch and descending aorta, and the patent true and false lumens (large arrow) at the level of the thoracoabdominal junction 
the majority of patients (i.e., with an acceptable body mass index) with lower limb arterial disease. For this purpose, they compared a group of 40 patients undergoing this CT regimen to a group of 40 patients undergoing CTA at $120 \mathrm{kVp}$ and $1.8 \mathrm{ml} / \mathrm{kg}$ contrast medium at the same rate as for the first group. The only significant difference between groups was a reduced contrast-to-noise ratio in the former. There was no intergroup difference in terms of arterial attenuation or in terms of qualitative visual scores. The most important fact was a reduction of $30 \%$ of both radiation dose and amount of contrast medium.

Decreasing the X-ray tube voltage decreases the amount of radiation used in the exam, and also decreases the average photon energy. For example, use of $100 \mathrm{kV}$ instead of $120 \mathrm{kV}$ reduces patient dose by a factor of 1.4. A comparison of the CTDIws at different tube voltages with 16-slice CTs from various vendors showed a range of $0.07-0.08 \mathrm{mGy}$ at $80 \mathrm{kV}$, $0.13-0.14 \mathrm{mGy}$ at $100 \mathrm{kV}, 0.16-0.22 \mathrm{mGy}$ at $120 \mathrm{kV}$, and $0.23-0.29$ at $140 \mathrm{kV}$ ! [9]. Furthermore, reducing the kilovoltage from 120 to $80 \mathrm{kV}$ leads to a $65 \%$ decrease in radiation dose at a constant tube current setting because the radiation dose varies with the square of the kilovoltage. Conversely, low kilovoltage CTA increases arterial enhancement because, as said in the study of Utsunomiya et al., contrast attenuation increases with reduced X-ray energy distribution due to the relatively high atomic number of iodine and the k-edge at such energy levels.

The reduction of the amount of contrast dose however needs careful attention to avoid outpacing of the bolus flow rate by $\mathrm{CT}$ data acquisition. Hence, the injection rate has to be adjusted so that the total injection time is appropriate for the data acquisition for each patient.

\section{Conclusion}

Diagnostic vascular imaging has continuously evolved since the advent of ultrasound, CT and MR. A new era of vascular imaging has emerged whereby non-invasive techniques have virtually replaced invasive techniques for diagnostic purposes. The recent improvements discussed here show that we should now be able to avoid, as much as possible, the classical complications of contrast media, as well as diminishing the potential radiation associated with diagnostic CT examinations. By virtue of these improvements, we can expect that diagnostic vascular imaging will become soon totally noninvasive. The radiological community must be aware of, and ready to share this fabulous adventure.

\section{References}

1. Krishnam MS, Tomasian A, Malik S, Desphande V, Laub G, Ruehm SG (2010) Image quality and diagnostic accuracy of unenhanced SSFP MR angiography compared with conventional contrast-enhanced MR angiography for the assessment of thoracic aortic diseases. Eur Radiol 20:1311-1320

2. Gjesdal KI, Storaas T, Geitung JT (2009) A noncontrast-enhanced pulse sequence optimized to visualize human peripheral vessels. Eur Radiol 19:110-120

3. Utsunomiya D, Oda S, Funama Y, Awai K, Nakaura T, Yanaga Y, Hirai T, Yamashita Y (2010) Comparison of standard- and low-tube voltage MDCT angiography in patients with peripheral arterial disease. Eur Radiol 20:2758-2765

4. Russo V, Renzulli M, Buttazzi K, Fattori R (2006) Acquired diseases of the thoracic aorta: role of MRI and MRA. Eur Radiol $16: 852-865$

5. Fuchs F, Laub G, Othomo K (2003) TrueFISP - technical considerations and cardiovascular applications. Eur J Radiol 46:28-32

6. Tomasian A, Lohan DG, Laub G, Singhal A, Finn JP, Krishnam MS (2008) Noncontrast 3D steady state free precession magnetic resonance angiography of the thoracic central veins using nonselective radiofrequency excitation over a large field of view: initial experience. Radiology 43:306-313

7. Krishnam MS, Tomasian A, Deshpande VS et al (2008) Non-contrast 3D SSFP MR angiography of the whole chest using non-selective RF excitation over a large field of view: comparison with single-phase 3D contrast-enhanced MRA. Invest Radiol 43:411-420

8. Brittain JH, $\mathrm{Hu} \mathrm{BS}$, Wright GA, Meyer $\mathrm{CH}$, Macovski A, Nishimura DG (1995) Coronary angiography with magnetizationprepared T2 contrast. Magn Reson Med 33:689-696

9. Huda W, Vance A (2007) Patient radiation doses from adult and pediatric CT. AJR Am J Roentgenol 188:540-546 